\title{
Safety and Effectiveness of Lenvatinib in 594 Patients with Unresectable Thyroid Cancer in an All-Case Post- Marketing Observational Study in Japan
}

\author{
Shunji Takahashi · Makoto Tahara - Koichi Ito · Masayuki Tori · \\ Naomi Kiyota $\cdot$ Katsutoshi Yoshida $\cdot$ Yukinori Sakata • \\ Akira Yoshida
}

Received: June 9, 2020 / Published online: July 16, 2020

(C) The Author(s) 2020

\section{ABSTRACT}

Introduction: Lenvatinib is approved in Japan for treating patients with all histological subtypes of unresectable thyroid cancer, including differentiated thyroid cancer (DTC), medullary thyroid cancer (MTC), and anaplastic thyroid cancer (ATC). However, safety and effectiveness

Digital Features To view digital features for this article go to https://doi.org/10.6084/m9.figshare.12588248.

Electronic Supplementary Material The online version of this article (https://doi.org/10.1007/s12325020-01433-8) contains supplementary material, which is available to authorized users.

S. Takahashi $(\bowtie)$

Department of Medical Oncology, Cancer Institute Hospital of the Japanese Foundation for Cancer

Research, 3-8-31, Ariake, Koto-ku, Tokyo 135-8550, Japan

e-mail: s.takahashi-chemotherapy@jfcr.or.jp

M. Tahara

Department of Head and Neck Cancer Oncology,

National Cancer Center Hospital East, Kashiwa,

Chiba 277-8577, Japan

K. Ito

Ito Hospital, 4-3-6 Jingu-mae, Shibuya-ku, Tokyo

150-8308, Japan

M. Tori

Department of Endocrine Surgery, Osaka Police Hospital, 10-31, Kitayamacho, Tennouji-ku, Osaka 543-0035, Japan data are limited in Japanese patients. Therefore, this prospective, post-marketing observational study evaluated, in daily clinical practice, the safety and effectiveness of lenvatinib in Japanese patients with unresectable thyroid cancer. Methods: All patients with unresectable thyroid cancer first treated with lenvatinib between May and November 2015 were registered. Patients were orally administered lenvatinib and followed up for 12 months. The endpoints included adverse drug reactions (ADRs), overall survival (OS), overall response rate (ORR), and time-to-treatment failure. Post hoc Cox multivariate analyses were performed to assess prognostic factors associated with the 12-month OS rate.

\section{N. Kiyota}

Oncology/Hematology, Kobe University Hospital Cancer Center, 7-5-1, Kusunokicho, Chuo-ku, Kobe, Hyogo 650-0017, Japan

K. Yoshida $\cdot$ Y. Sakata

Eisai Co. Ltd., 4-6-10 Koishikawa, Bunkyo-ku, Tokyo 112-8088, Japan

A. Yoshida

Kanagawa Health Service Association, Nihon-odori bldg. 58 Nihon-odori, Naka-ku, Yokohama 2310021, Japan 
Results: Of 629 registered patients, 594 were included in the analysis. A total of 442 patients (74.4\%) had DTC, 28 (4.7\%) had MTC, and 124 (20.9\%) had ATC. Hypertension, proteinuria, and palmar-plantar erythrodysesthesia syndrome were the most frequently reported ADRs across all histological subtypes. The median OS was 101.0 days in patients with ATC which was not reached in patients with DTC and patients with MTC, with 12 -month OS rates of $15.6 \%$, $75.7 \%$, and $83.0 \%$, respectively. The ORRs were $59.2 \%, 45.0 \%$, and $43.8 \%$ among 368 patients with DTC, 20 with MTC, and 105 with ATC, respectively. Multivariate analyses revealed that Eastern Cooperative Oncology Group performance status (ECOG PS), tumor size, the presence of tumor invasion, and body weight were baseline prognostic factors affecting OS in patients with DTC, while ECOG PS and the presence of liver metastasis were prognostic factors in patients with ATC.

Conclusion: Lenvatinib demonstrated an acceptable safety profile for patients with thyroid cancer in a real-world setting in Japan. The safety profile and effectiveness findings for lenvatinib in this study were consistent with those from previous clinical trials, irrespective of histological subtype.

Keywords: Effectiveness; Lenvatinib; Observational study; Safety; Tyrosine kinase inhibitor

\section{Key Summary Points}

Why carry out this study?

Lenvatinib, an oral, multitargeted tyrosine kinase inhibitor, was approved for the treatment of patients with unresectable thyroid cancer of all histological subtypes in Japan.

The efficacy and safety data for lenvatinib is limited to clinical trials conducted prior to approval in Japan.
This all-case post-marketing observational study was conducted to assess safety and effectiveness of lenvatinib in patients with thyroid cancer, including differentiated thyroid cancer (DTC), medullary thyroid cancer (MTC), and anaplastic thyroid cancer (ATC).

\section{What was learned from the study?}

Acceptable safety profile and meaningful antitumor activities of lenvatinib in patients with unresectable thyroid cancer-including 124 patients with ATC - in the real-world clinical setting was indicated, irrespective of the histological subtype.

The antitumor activity and tolerability of lenvatinib are of benefit to patients with unresectable thyroid cancer and can be added to the limited treatment options available for such patients.

\section{INTRODUCTION}

Thyroid cancer is a common malignant endocrine tumor that is classified into three main histological subtypes: differentiated thyroid cancer (DTC), medullary thyroid cancer (MTC), and anaplastic thyroid cancer (ATC) [1]. Among these histological subtypes, DTC is the most common, accounting for more than $97 \%$ of thyroid cancer cases in Japan; MTC and ATC account for less than $2 \%$ of cases each [2]. Although the prognosis of patients with DTC is optimistic, with a 10-year survival rate exceeding 90\% [3], the presence or development of distant metastasis and resistance to radioactive iodine (RAI) results in a poor prognosis $[4,5]$. The 10-year survival rate after the detection of metastasis was only $10 \%$ in patients with radioactive iodine-refractory (RR)-DTC [6]; and in patients with rapidly progressive lung metastasis (average tumor volume doubling time of at most 1 year), the 5 -year survival was $19 \%$ after pulmonary nodules exceeded $1 \mathrm{~cm}$ in diameter [7]. In addition, the 10-year survival rate for patients with MTC and distant 
metastases at diagnosis was only $40 \%$ [8]. Among patients with ATC, the median survival time after diagnosis was 3-4 months [9] and the 1 -year survival rate was approximately $18-20 \%$ $[9,10]$.

Lenvatinib is an oral, multitargeted tyrosine kinase inhibitor of the vascular endothelial growth factor (VEGF) receptors 1-3, fibroblast growth factor receptors 1-4, platelet-derived growth factor receptor- $\alpha$, RET, and KIT signaling networks, all of which have been implicated in tumor angiogenesis [11-13]. The findings of the randomized, double-blind, placebo-controlled, phase III SELECT trial indicated that treatment with lenvatinib prolonged the progression-free survival of patients with RR-DTC $[14,15]$. Lenvatinib was approved in many countries including the USA and EU for the treatment of locally recurrent or metastatic, progressive RRDTC. Following the results of a phase II trial in Japanese patients with RR-DTC, MTC, and ATC [16], lenvatinib was approved for the treatment of patients with unresectable thyroid cancer of all histological subtypes in Japan.

The approval for lenvatinib in Japan was given on the basis of the efficacy and safety data in a limited number of patients with thyroid cancer, i.e., a total of 81 patients, of which only 17 patients had ATC. Therefore, as a requirement for the approval of lenvatinib by the Japanese Pharmaceutical and Medical Devices Agency, an all-case post-marketing observational study was conducted to assess the safety and effectiveness of lenvatinib in Japanese patients with thyroid cancer, including DTC, MTC, and ATC, in a clinical setting. In addition, we determined the prognostic factors of survival in these patients.

\section{METHODS}

\section{Study Design}

The current study was an all-case, post-marketing observational study of lenvatinib (Lenvima ${ }^{\circledR}$ ) for patients with unresectable thyroid cancer (ClinicalTrials.gov Trial Registration ID NCT02430714). Patients were enrolled between May 2015 (launch of Lenvima ${ }^{\circledR}$ ) and November
2015. This study was performed in accordance with the guidelines given in the Declaration of Helsinki as well as the Pharmaceutical Affairs Law and the ministerial ordinance of Good Post-Marketing Study Practice (GPSP) in Japan.

\section{Patients}

All patients with unresectable thyroid cancer who were administered lenvatinib for the first time in Japan were centrally registered at the time of first administration of lenvatinib, and the safety and effectiveness of lenvatinib were monitored for 1 year. Among patients whose treatment was completed or discontinued within 1 year after lenvatinib administration, the survival status (dead or alive) was documented at the end of the observational period. Among those who were lost to follow-up, owing to reasons such as death or being transferred to another hospital, the observational period ended at the time that the patient was lost to follow-up.

\section{Treatments}

Lenvatinib $24 \mathrm{mg}$ was orally administered once daily in accordance with the package insert. The dose was reduced, or treatment was interrupted or discontinued at the discretion of the attending physicians.

\section{Assessments}

\section{Assessment of Safety}

Adverse events (AEs) were recorded from the first lenvatinib administration to 14 days after the completion or discontinuation of lenvatinib treatment. AEs which could not be ruled out as being caused by lenvatinib at the discretion of the attending physicians were categorized as adverse drug reactions (ADRs). ADRs were classified according to the Medical Dictionary for Regulatory Activities (MedDRA), version 20.1, and their severities were graded according to the Japanese version of the Common Terminology Criteria for Adverse Events, version 4.0. ADRs of interest included hypertension; hemorrhagic events in addition to the following: carotid 
artery hemorrhage, venous hemorrhage, and tumor hemorrhage associated with tumor shrinkage or necrosis; thromboembolic events; hepatic disorders; renal disorders; perforation of the digestive tract and gastrointestinal fistula; reversible posterior leukoencephalopathy syndrome; cardiac dysfunction; arrhythmia; palmar-plantar erythrodysesthesia syndrome; infectious diseases; hematological toxicity; hypocalcemia; protracted wound healing; and increased blood-thyroid-stimulating hormone level.

\section{Assessment of Effectiveness}

Data were collected at 3, 6, and 12 months after the first administration of lenvatinib. Overall survival (OS) was defined as the time from the first administration of lenvatinib until death from any cause, or the last date the patient was known to be alive (censored). The 12-month OS rate was determined as the proportion of patients who were alive at 12 months. The timeto-treatment failure (TTF) was defined as the time from the first administration of lenvatinib to the date of treatment discontinuation owing to any cause (e.g., disease progression, AEs, patient request, or death). Patients in whom treatment was continued for 12 months after the first administration of lenvatinib were censored at the last administration of lenvatinib.

Tumor responses for the single largest tumor were evaluated over 12 months after the administration of lenvatinib, using imaging data including computed tomography (CT). The best overall response was classified into the following five categories: complete response (CR), which indicated disappearance of the tumor; partial response (PR), which indicated at least a 30\% decrease in the tumor diameter, taking as reference the baseline diameter; stable disease (SD); progressive disease (PD), which indicated at least a $20 \%$ increase in the tumor diameter, taking as reference the baseline diameter; and not evaluable (NE). Unlike the Response Evaluation Criteria in Solid Tumors (RECIST) guidelines, tumors in the current study did not need to show CR and PR at 4 weeks or more to be indicators of those responses. In fact, tumor responses were recorded at the discretion of the attending physician and were not reviewed by the independent central review board. The overall response rate (ORR) was defined as the proportion of patients with CR and PR.

\section{Statistical Analysis}

All analyses were conducted for each of the three histological subtypes: DTC, MTC, and ATC. The patient demographic and baseline characteristics, administration dose of lenvatinib, frequency of ADRs of interest, and frequency of ADRs of at least grade 3 were descriptively analyzed. The Kaplan-Meier method was used to estimate the median OS (days), TTF (days), and the 12-month OS rate along with the corresponding 95\% confidence intervals $(\mathrm{CI})$.

Prognostic factors affecting OS were also assessed using univariate and multivariate Cox regression analyses. The hazard ratio (HR) for each factor and its 95\% CI were estimated. After the univariate analysis, all factors were entered into a multivariate stepwise model with selection criteria of $p<0.20$. These analyses were not conducted for patients with MTC owing to the small sample size.

A statistical significance level of $p<0.05$ was used for the remaining analyses. All statistical analyses were performed using Statistical Analysis System (SAS) Release 9.4 (SAS Institute Inc., Cary, NC, USA).

\section{RESULTS}

\section{Patients}

A total of 629 patients were registered between May and November 2015 at 268 institutions. Of those, the case report forms of 628 patients were collected. After exclusion of 24 patients who were identified as duplicate registration cases that were transferred to another hospital and 10 patients who did not meet the criteria of the three histological subtypes (DTC, MTC, and ATC), a total of 594 patients were included in the analysis. Of these, 442 patients (74.4\%) had 
Table 1 Demographic and baseline characteristics of patients according to the histological subtypes

\begin{tabular}{|c|c|c|c|}
\hline & DTC $(n=442)$ & MTC $(n=28)$ & $\operatorname{ATC}(n=124)$ \\
\hline Age, years (median, range) & $70.0(19-90)$ & $63.0(43-85)$ & $73.0(41-88)$ \\
\hline Female sex & $266(60.2)$ & $10(35.7)$ & $76(61.3)$ \\
\hline Body weight, $\mathrm{kg}$ (mean, SD) & $55.6(12.4)$ & $56.8(12.9)$ & $51.7(12.1)$ \\
\hline \multicolumn{4}{|l|}{ ECOG PS score } \\
\hline 0 & $224(50.7)$ & $17(60.7)$ & $38(30.6)$ \\
\hline 1 & $159(36.0)$ & $10(35.7)$ & $50(40.3)$ \\
\hline 2 & $35(7.9)$ & $1(3.6)$ & $18(14.5)$ \\
\hline 3 & $20(4.5)$ & $0(0.0)$ & $13(10.5)$ \\
\hline 4 & $4(0.9)$ & $0(0.0)$ & $5(4.0)$ \\
\hline Time from the first onset of thyroid cancer, years (median, range) & $7.83(0.1-62.5)$ & $8.50(0.3-34.9)$ & $0.42(0.0-25.4)$ \\
\hline \multicolumn{4}{|l|}{ Histological subtype } \\
\hline Papillary carcinoma & $323(73.1)$ & - & - \\
\hline Follicular carcinoma & $62(14.0)$ & - & - \\
\hline Papillary and follicular carcinoma & $1(0.2)$ & - & - \\
\hline Poorly differentiated & $56(12.7)$ & - & - \\
\hline Sites of metastasis ${ }^{a}$ & 389 & 23 & 92 \\
\hline Lungs & $342(87.9)$ & $10(43.5)$ & $71(77.2)$ \\
\hline Distant nodes & $85(21.9)$ & $5(21.7)$ & $24(26.1)$ \\
\hline Bones & $145(37.3)$ & $12(52.2)$ & $18(19.6)$ \\
\hline Brain & $19(4.9)$ & $0(0.0)$ & $1(1.1)$ \\
\hline Liver & $24(6.2)$ & $12(52.2)$ & $16(17.4)$ \\
\hline Others & $49(12.6)$ & $0(0.0)$ & $15(16.3)$ \\
\hline History of surgery & $419(94.8)$ & $25(89.3)$ & $66(53.2)$ \\
\hline \multicolumn{4}{|l|}{ History of chemotherapy } \\
\hline Yes & $149(33.7)$ & $6(21.4)$ & $39(31.5)$ \\
\hline No & $292(66.1)$ & $22(78.6)$ & $85(68.5)$ \\
\hline Unknown & $1(0.2)$ & $0(0.0)$ & $0(0.0)$ \\
\hline \multicolumn{4}{|l|}{ History of TSH suppression therapy } \\
\hline Yes & $306(69.2)$ & $10(35.7)$ & $35(28.2)$ \\
\hline No & $123(27.8)$ & $16(57.1)$ & $87(70.2)$ \\
\hline Unknown & $13(2.9)$ & $2(7.1)$ & $2(1.6)$ \\
\hline \multicolumn{4}{|l|}{ Postoperative ${ }^{131} \mathrm{I}$ ablation } \\
\hline Administered & $142(32.1)$ & - & - \\
\hline
\end{tabular}


Table 1 continued

\begin{tabular}{|c|c|c|c|}
\hline & DTC $(n=442)$ & MTC $(n=28)$ & $\operatorname{ATC}(n=124)$ \\
\hline Not administered & $275(62.2)$ & - & - \\
\hline Unknown & $25(5.7)$ & - & - \\
\hline \multicolumn{4}{|c|}{ Radioisotope therapy with ${ }^{131} \mathrm{I}$ for metastasis } \\
\hline Administered & $281(63.6)$ & - & - \\
\hline Not administered & $153(34.6)$ & - & - \\
\hline Unknown & $8(1.8)$ & - & - \\
\hline \multicolumn{4}{|c|}{ Treatment with postoperative ${ }^{131} \mathrm{I}$ ablation or radioisotope therapy with ${ }^{131} \mathrm{I}$ for metastasis } \\
\hline Administered & $326(73.8)$ & - & - \\
\hline Not administered & $111(25.1)$ & - & - \\
\hline Unknown & $5(1.1)$ & - & - \\
\hline
\end{tabular}

The total might not equal $100 \%$ owing to rounding. Data are expressed as the number and percentage unless specified otherwise

$D T C$ differentiated thyroid cancer, $M T C$ medullary thyroid cancer, $A T C$ anaplastic thyroid cancer, ECOG PS Eastern Cooperative Oncology Group performance status, TSH thyroid-stimulating hormone

${ }^{\text {a }}$ Sites of metastasis include multiple responses

DTC, 28 (4.7\%) had MTC, and 124 (20.9\%) had ATC.

\section{Patient Baseline Characteristics}

The patient demographics and baseline characteristics according to each subtype are shown in Table 1 . The median age and sex distribution in patients with DTC and patients with ATC were similar (DTC: 70.0 years [range 19-90 years] with $60.2 \%$ women; ATC: 73.0 years [range $41-88$ years], with $61.3 \%$ women); patients with MTC were relatively younger (median age 63.0 years [range $43-85$ years]) and included fewer women (35.7\%). Most patients had Eastern Cooperative Oncology Group performance status (ECOG PS) scores of at most $1(86.7 \%$ of patients with DTC, $96.4 \%$ of patients with MTC, and $71.0 \%$ of patients with ATC). The most common site of distant metastasis was the lungs in patients with DTC and patients with ATC (87.9\% and $77.2 \%$, respectively), whereas it was the bones and the liver in patients with MTC (52.2\% for both locations). The majority of patients with DTC and patients with MTC had a history of surgical treatment $(94.8 \%$ and $89.3 \%$, respectively), while $53.2 \%$ of patients with ATC had undergone surgery. A total of $28.5 \%$ patients with DTC, $7.1 \%$ patients with MTC, and $19.4 \%$ patients with ATC were previously treated with sorafenib, vandetanib, and weekly paclitaxel, respectively.

Among 442 patients with DTC, $73.8 \%$ received postoperative ${ }^{131} \mathrm{I}$ ablation or radioisotope therapy with ${ }^{131} \mathrm{I}$ for metastasis (Table 1).

\section{Lenvatinib Administration: Dose Exposure and Discontinuation}

Most patients received lenvatinib at a dose at $24 \mathrm{mg}$ once daily regardless of the subtypes (76.2\% of DTC, $78.6 \%$ of MTC, and $70.2 \%$ of patients with ATC; Table 2). The daily mean dose during the study period was $12.06 \pm 5.46 \mathrm{mg}$ in patients with DTC, $12.72 \pm 5.60 \mathrm{mg}$ in patients with MTC, and $15.71 \pm 6.36 \mathrm{mg}$ in patients with ATC. 
Table 2 Administration dose of lenvatinib according to the histological subtypes

\begin{tabular}{llll}
\hline & DTC $(n=4 \mathbf{4 2})$ & MTC $(\boldsymbol{n}=\mathbf{2 8})$ & ATC $(\boldsymbol{n}=\mathbf{1 2 4})$ \\
\hline Initial dose per day & & & \\
$24 \mathrm{mg}$ & $337(76.2)$ & $22(78.6)$ & $87(70.2)$ \\
$20 \mathrm{mg}$ & $29(6.6)$ & $2(7.1)$ & $18(14.5)$ \\
$14 \mathrm{mg}$ & $29(6.6)$ & $0(0.0)$ & $9(7.3)$ \\
$10 \mathrm{mg}$ & $26(5.9)$ & $4(14.3)$ & $6(4.8)$ \\
$8 \mathrm{mg}$ & $15(3.4)$ & $0(0.0)$ & $2(1.6)$ \\
$4 \mathrm{mg}$ & $0(0.0)$ & $0(0.0)$ & $0(0.0)$ \\
Others & $6(1.4)$ & $0(0.0)$ & $2(1.6)$ \\
Mean dose, mg/day, $\pm \mathrm{SD}^{\mathrm{b}}$ & $12.06 \pm 5.46$ & $12.72 \pm 5.60$ & $15.71 \pm 6.36$ \\
\hline
\end{tabular}

The total might not equal $100 \%$ owing to rounding. Data are expressed as the number and percentage unless specified otherwise

$D T C$ differentiated thyroid cancer, $M T C$ medullary thyroid cancer, $A T C$ anaplastic thyroid cancer, $S D$ standard deviation

${ }^{a}$ Others include $18 \mathrm{mg}, 16 \mathrm{mg}$, and $12 \mathrm{mg}$

b The mean dose was calculated by cumulating the lenvatinib dose administered during the study period divided by the administration days

\section{Assessment of Safety}

ADRs were experienced by $100 \%$ of MTC, $98.2 \%$ of DTC, and $92.7 \%$ of patients with ATC (Table 3). The most frequently reported major ADRs were hypertension, proteinuria, and palmar-plantar erythrodysesthesia syndrome, regardless of the histological subtype of thyroid cancer. The following ADRs of at least grade 3 had an incidence of greater than 5\%: hypertension $(60.2 \%)$, proteinuria $(17.2 \%)$, and palmar-plantar erythrodysesthesia syndrome (5.9\%) among patients with DTC; hypertension $(39.3 \%)$, proteinuria $(14.3 \%)$, and diarrhea (10.7\%) among patients with MTC; and hypertension $(46.8 \%)$, platelet count decreased (12.9\%), and proteinuria (7.3\%) among patients with ATC (Table 3). Of fatal AEs experienced within 30 days after the last administration of lenvatinib in 52 patients with DTC (11.8\%), 1 patient with MTC $(3.6 \%)$, and 75 patients with ATC $(60.5 \%)$, fatal ADRs occurred in $2.7 \%$ of patients with DTC, $3.6 \%$ of patients with MTC, and $4.8 \%$ of patients with ATC (Table S1).

The 16 ADRs of interest are shown in Table S2. Hypertension was the most frequently reported ADR across the three subtypes (79.4\% of patients with DTC, $64.3 \%$ of patients with MTC, and $70.2 \%$ of patients with ATC). A total of $1.6 \%$ and $8.1 \%$ of patients with DTC and patients with ATC, respectively, showed carotid artery hemorrhage, venous hemorrhage, and tumor hemorrhage associated with tumor shrinkage or necrosis, while none of the patients with MTC showed these ADRs (Table S2).

ADRs that led to treatment discontinuation in at least $2 \%$ of all patients were proteinuria $(4.6 \%)$, decreased appetite (3.3\%), malaise (2.5\%), hypertension (2.3\%), and platelet count decreased $(2.0 \%)$. No notable differences in ADRs were reported between patients with and without a history of RAI treatment (Table S3).

\section{Effectiveness}

The lenvatinib effectiveness results are shown in Table 4. The median OS was 101.0 days (i.e., 3.3 months) in patients with ATC, and was not reached in patients with DTC and patients with MTC (Fig. 1). The 12-month OS rate was 75.7\% in patients with DTC, $83.0 \%$ in patients with MTC, and $15.6 \%$ in patients with ATC. The median TTF was 375.0 days in patients with DTC, 301.0 days in patients with MTC, and 
Table 3 Adverse drug reactions according to the histological subtypes

\begin{tabular}{|c|c|c|c|c|c|c|}
\hline \multirow[b]{2}{*}{ Any ADR, $n(\%)$} & \multicolumn{2}{|c|}{ DTC $(n=442)$} & \multicolumn{2}{|c|}{ MTC $(n=28)$} & \multicolumn{2}{|c|}{$\operatorname{ATC}(n=124)$} \\
\hline & $434(98.2)$ & & $28(100.0)$ & & $115(92.7)$ & \\
\hline $\mathrm{ADR}, n(\%)$ & Any grade & Grade $\geq 3$ & Any grade & Grade $\geq 3$ & Any grade & Grade $\geq 3$ \\
\hline Hypertension $^{\mathrm{a}}$ & $353(79.4)$ & $267(60.2)$ & $18(64.3)$ & $11(39.3)$ & $87(70.2)$ & $58(46.8)$ \\
\hline Proteinuria & $188(42.5)$ & $76(17.2)$ & $11(39.3)$ & $4(14.3)$ & $37(29.8)$ & $9(7.3)$ \\
\hline $\begin{array}{l}\text { Palmar-plantar erythrodysesthesia } \\
\text { syndrome }^{\text {b }}\end{array}$ & $174(39.1)$ & $26(5.9)$ & $14(50.0)$ & $1(3.6)$ & $32(25.8)$ & $4(3.2)$ \\
\hline Decreased appetite & $115(26.0)$ & $20(4.5)$ & $7(25.0)$ & $1(3.6)$ & $20(16.1)$ & $6(4.8)$ \\
\hline Platelet count decreased & 97 (21.9) & $20(4.5)$ & $4(14.3)$ & $1(3.6)$ & $28(22.6)$ & $16(12.9)$ \\
\hline Malaise & $88(19.9)$ & $10(2.3)$ & $3(10.7)$ & $0(0.0)$ & $24(19.4)$ & $1(0.8)$ \\
\hline Diarrhea & $78(17.6)$ & $17(3.8)$ & $7(25.0)$ & $3(10.7)$ & $15(12.1)$ & $1(0.8)$ \\
\hline
\end{tabular}

$D T C$ differentiated thyroid cancer, $M T C$ medullary thyroid cancer, $A T C$ anaplastic thyroid cancer, $A D R$ adverse drug reactions

ADRs were categorized on the basis of the preferred terms of the Medical Dictionary for Regulatory Activities (MedDRA), version 20.1

An ADR was counted as one event when the identical ADR was reported multiple times in 1 patient

${ }^{a}$ Hypertension included the MedDRA preferred terms of blood pressure diastolic increased, blood pressure increased, diastolic hypertension, hypertension, systolic hypertension, and prehypertension

b Palmar-plantar erythrodysesthesia included the MedDRA preferred terms of erythema multiforme, palmar-plantar erythrodysesthesia syndrome, palmar erythema, rash erythematous, skin reaction, and hand dermatitis

74.5 days in patients with ATC (i.e., 12.3 months, 9.9 months, 2.4 months, respectively). The incidence of treatment continuation for 12 months was highest in patients with DTC $(52.2 \%)$, followed by patients with MTC (48.1\%), and patients with ATC (9.2\%).

Tumor response was assessed among patients whose tumor response data was collected and available which was $83.3 \%, 71.4 \%$, and $84.7 \%$ of patients with DTC, MTC, and ATC, respectively. The ORR was $59.2 \%$ in patients with DTC, $45.0 \%$ in patients with MTC, and $43.8 \%$ in patients with ATC (Table 4). The disease control rate (DCR) was $92.4 \%$ in patients with DTC, $100.0 \%$ in patients with MTC, and $76.2 \%$ in patients with ATC.

\section{Prognostic Factors Affecting OS of Patients with DTC and Those with ATC after Lenvatinib Administration}

The results of the univariate analysis are shown in Fig. S1a, b. Multivariate Cox regression analysis revealed the following baseline prognostic factors affecting OS: body weight, ECOG PS score, tumor diameter prior to lenvatinib administration, and the presence of tumor invasion to the carotid artery, jugular artery, trachea, skin, or other region in patients with DTC (Fig. S2a). The ECOG PS score and the presence of liver metastasis were significantly associated with the OS of patients with ATC (Fig. S2b).

\section{DISCUSSION}

In the current all-case post-marketing observational study, we assessed the safety and effectiveness of lenvatinib in 594 Japanese patients with unresectable thyroid cancer. The results demonstrated the tolerability and meaningful antitumor activity of lenvatinib, irrespective of the histological subtype.

The current study included patients with various demographic and baseline 
Table 4 Assessment of the effectiveness of lenvatinib according to the histological subtypes

\begin{tabular}{llll}
\hline & DTC & MTC & ATC \\
\hline$N$ & 442 & 28 & 124 \\
Overall survival, days, median (95\% CI) & - & - & $101.0(80.0-130.0)$ \\
12-month overall survival rate, \% (95\% CI) & $75.7(71.3-79.5)$ & $83.0(60.7-93.3)$ & $15.6(9.6-22.9)$ \\
Time-to-treatment failure, days, median (95\% CI) & $375.0(302.0-511.0)$ & $301.0(198.0-405.0)$ & $74.5(57.0-108.0)$ \\
12-month treatment continuation, \% (95\% CI) & $52.2(47.0-57.1)$ & $48.1(26.5-66.7)$ & $9.2(4.8-15.5)$ \\
$N$ & 368 & 20 & 105 \\
Best overall response ${ }^{\mathrm{a}}, n$ (\%) & & & \\
Complete response & $10(2.7)$ & $1(5.0)$ & $3(2.9)$ \\
Partial response & $208(56.5)$ & $8(40.0)$ & $43(41.0)$ \\
Stable disease & $122(33.2)$ & $11(55.0)$ & $34(32.4)$ \\
Progressive disease & $25(6.8)$ & $0(0.0)$ & $25(23.8)$ \\
Not evaluable & $3(0.8)$ & $0(0.0)$ & $0(0.0)$ \\
Overall response rate $^{\mathrm{b}}, \%$ (95\% CI) & $59.2(54.03-64.30)$ & $45.0(23.06-68.47)$ & $43.8(34.14-53.83)$ \\
Disease control rate $^{\mathrm{c}}, \%$ (95\% CI) & $92.4(89.19-94.88)$ & $100.0(83.16-100.00)$ & $76.2(66.89-83.96)$ \\
\hline
\end{tabular}

$D T C$ differentiated thyroid cancer, $M T C$ medullary thyroid cancer, $A T C$ anaplastic thyroid cancer, $C I$ confidence interval

a Tumor responses for the single largest tumor were evaluated using imaging data including computed tomography (CT), and the best response during 12 months after the first administration of lenvatinib was recorded as best overall response. The responses were classified into complete response (CR), which indicated a disappearance of the tumor; partial response $(\mathrm{PR})$, which indicated $\mathrm{a} \geq 30 \%$ decrease in the tumor diameters, taking as reference the baseline diameter; stable disease $(\mathrm{SD})$; progressive disease $(\mathrm{PD})$, which indicated $\mathrm{a} \geq 20 \%$ increase in the diameters, taking as reference the baseline diameter; and not evaluable (NE). Unlike the Response Evaluation Criteria in Solid Tumors (RECIST) guidelines, tumors in the current study did not need to show CR and PR at $\geq 4$ weeks to be indicators of those responses. Tumor responses were recorded at the discretion of the attending physician and were not reviewed by an independent central review board b The overall response rate indicates the sum of the proportion of patients with CR and those with PR

c The disease control rate indicates the sum of the proportion of patients with CR, PR, and SD

characteristics. Compared to the previous Japanese phase II and Japanese and global phase III SELECT studies [14-16], the current study included patients with more advanced age and a poorer performance status, as well as patients with DTC who were not treated with RAI after surgery. These differences were probably because patients were included from Japanese real-world clinical settings. It should be noted that the older age and poorer performance status of patients could have influenced the safety and effectiveness results to some extent in the current study, considering that higher toxicity of lenvatinib was observed in older patients [17].
The safety profile of lenvatinib in this postmarketing observational study was consistent overall with those observed in previous studies. Similar to the results of the previous phase III SELECT trial in which all the Japanese patients experienced ADRs [15], ADRs were observed in almost all patients in the current study. Although the incidence of ADRs of interest was lower overall in the current study, hypertension, proteinuria, and palmar-plantar erythrodysesthesia were the most frequently reported ADRs in patients with DTC, MTC, and ATC, similar to that reported in the previous trial [15]. Hypertension and proteinuria are the main AEs of VEGF-targeted therapies, as 


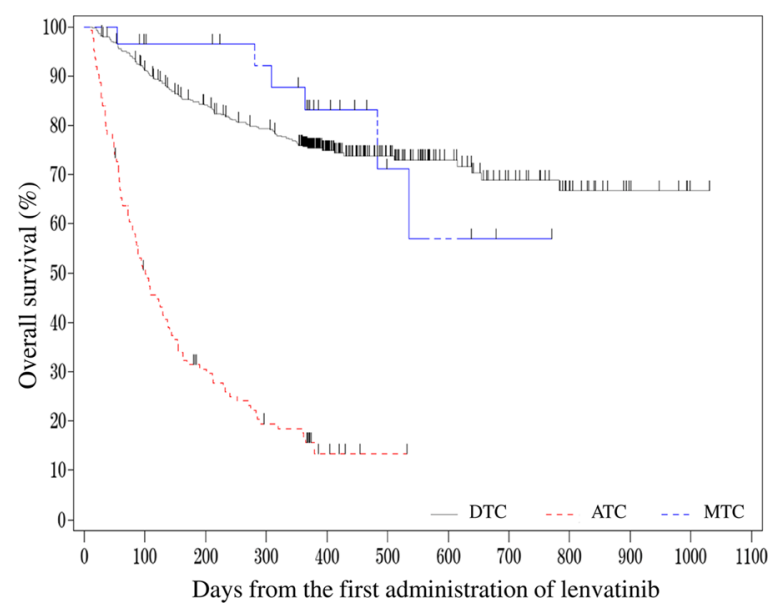

Fig. 1 Overall survival curve of patients with DTC, MTC, and ATC. DTC differentiated thyroid cancer, MTC medullary thyroid cancer, ATC anaplastic thyroid cancer

reported previously [18-20]. As patients with hypertension and proteinuria who discontinued treatment accounted for only small proportions, regardless of the high incidence of these ADRs of at least grade 3 in the current study, the appropriate management of these toxicities in a clinical setting resulted in lower rates of treatment discontinuation. Consequently, no new safety concern was observed except hemorrhage. Importantly, hemorrhage associated with tumor shrinkage or necrosis was higher in patients with ATC than in patients with DTC and patients with MTC (8.1\% vs. $1.6 \%$ and $0.0 \%$, respectively). Among patients with ATC, more careful monitoring and administration of lenvatinib are needed for patients with tumors that grow rapidly or tumors that metastasize close to the carotid artery, jugular artery, or hilus, as suggested previously regarding treatment with multitargeted kinase inhibitors including lenvatinib [21-23].

The effectiveness results of lenvatinib demonstrated overall a meaningful antitumor activity and survival rates that were comparable with those of previous trials. In the current study, the ORRs were $59.2 \%, 45.0 \%$, and $43.8 \%$ for patients with DTC, MTC, and ATC, respectively. Although the median OS could not be reached, possibly because the follow-up for this study was limited to 1 year, the 12-month os rate among patients with DTC (75.7\%) in the current study was comparable with that of patients with RR-DTC $(81.6 \%)$ in the previous global SELECT trial [14]. In contrast, the median OS of patients with ATC was shorter than that of patients in the phase II trial (10.6 months) [16]. This may be attributable to differences in the distribution of ECOG PS scores between the two studies: patients with ATC with a score of 0 and at least 3 accounted for $30.6 \%$ and $14.5 \%$, respectively, in the current study, while the corresponding rates in the previous trial were $59 \%$ and $0 \%$ as the previous trial included patients with an ECOG PS score of at most 2 [16].

The prognostic factors affecting the OS of patients with DTC included body weight before lenvatinib administration, ECOG PS, tumor size, and the presence of tumor invasion to the carotid artery, jugular artery, trachea, skin, or other region. The results of the current study were mostly consistent with the results of previous sub-analysis of the phase III SELECT trial that showed those with an ECOG PS score of 0 had a longer OS than those with an ECOG PS score of 1 [24]. Previous studies also showed that both invasion, such as vascular invasion, [25-28] and tumor size [3, 28-30] were prognostic factors for patients with DTC, including RR-DTC, reflecting the biological aggressiveness of tumors. Among patients with ATC, ECOG PS and the presence of liver metastasis were prognostic factors affecting OS in the current study. In previous studies, distant metastasis [9, 31, 32] was a prognostic factor among patients with ATC. The administration of lenvatinib among patients with ATC and liver metastasis may not be beneficial for their prognosis given that the median OS was 39.5 days and the 1-year OS rate was $0 \%$ among 16 patients in our univariate Cox regression results. Therefore, along with previous findings in the phase III SELECT trial, our findings suggest that the administration of lenvatinib before the appearance of any condition associated with poor prognosis may prolong the survival of DTC as well as patients with ATC.

The current study has several limitations. The data regarding histological subtype were collected on the basis of the information given 
by the investigator at each study site. The tumor assessment was not performed for $16.7 \%$, $28.6 \%$, and $15.3 \%$ of patients with DTC, MTC, and ATC, respectively. Moreover, the assessment was performed at the attending physician's discretion, and the result was not reviewed at an independent central review board of physicians. Therefore, the results regarding the effectiveness of lenvatinib might have been somewhat overestimated or underestimated. The current results were discussed in comparison with the previous phase II and SELECT studies of lenvatinib, which included patients with DTC limited to those who were refractory to RAI therapy, whereas this study partly included patients with DTC whose RAIrefractory status was not confirmed. Thus, direct comparison of the results may not be plausible. Finally, because the data regarding safety were collected for only 12 months, further studies are needed to examine the long-term safety of lenvatinib.

\section{CONCLUSIONS}

The current study was the first to evaluate the safety and effectiveness of lenvatinib in a large number of patients with unresectable thyroid cancer-including 124 patients with ATC-after its approval in Japan. Our results indicated an acceptable safety profile and meaningful antitumor activities of lenvatinib in Japanese patients with unresectable thyroid cancer in a real-world clinical setting, irrespective of the histological subtype. The antitumor activity and tolerability of lenvatinib demonstrated in the current real-world study are of benefit for patients with unresectable thyroid cancer, including DTC, MTC, and ATC, as lenvatinib can be added to the limited treatment options available for such patients.

\section{ACKNOWLEDGEMENTS}

Funding. This work was supported by Eisai Co. Ltd., Tokyo, Japan. Eisai Co. Ltd. also funded the journal's Rapid Service and Open Access Fees.

Editorial Assistance. Editorial assistance in the preparation of this article was provided by Mika Kawaguchi, Clinical Study Support, Inc., Nagoya, Japan, under contract with Eisai Co. Ltd.

Authorship. All named authors meet the International Committee of Medical Journal Editors (ICMJE) criteria for authorship for this article, take responsibility for the integrity of the work as a whole, and have given their approval for this version to be published.

Disclosures. S Takahashi received honoraria from Eisai, Novartis, Taiho, MSD, Chugai, Daiichi-Sankyo, Bayer, and AstraZeneca. M Tahara received honoraria from Bayer, Bristol Myers Squibb, Eisai, Merck Serono, and Takeda; received consulting or advisory fees from Bayer, Boehringer-Ingelheim, Bristol Myers Squibb, MSD, Ono Pharmaceutical, and Pfizer; and received research funding from AstraZeneca, Bayer, Boehringer-Ingelheim, Eisai, Merck Sharp \& Dohme, NanoCarrier, Novartis, Ono Pharmaceutical, and Pfizer. N Kiyota received honoraria from Ono Pharmaceutical, Bristol Myers Squibb, Merck Serono, AstraZeneca, Eisai, and Bayer; and received research funding from Eisai, AstraZeneca, Pfizer, Chugai Pharmaceutical, Ono Pharmaceutical, and Bristol-Myers Squibb. K Yoshida and Y Sakata are employees of Eisai. A Yoshida is a medical advisor of Eisai. $\mathrm{K}$ Ito and $\mathrm{M}$ Tori have nothing to disclose.

Compliance with Ethics Guidelines. This study was conducted in accordance with the Declaration of Helsinki and Japanese Good Post-Marketing Study Practice (GPSP), an authorized standard for post-marketing surveillance. Obtaining ethical approval from an institutional review board or consent from participating patients is not required for GPSP. Personal data related to this study were managed in compliance with the privacy protection laws in Japan. 
Data Availability. The datasets generated during and/or analyzed during the current study are available from the corresponding author on reasonable request.

Open Access. This article is licensed under a Creative Commons Attribution-NonCommercial 4.0 International License, which permits any non-commercial use, sharing, adaptation, distribution and reproduction in any medium or format, as long as you give appropriate credit to the original author(s) and the source, provide a link to the Creative Commons licence, and indicate if changes were made. The images or other third party material in this article are included in the article's Creative Commons licence, unless indicated otherwise in a credit line to the material. If material is not included in the article's Creative Commons licence and your intended use is not permitted by statutory regulation or exceeds the permitted use, you will need to obtain permission directly from the copyright holder. To view a copy of this licence, visit http://creativecommons.org/licenses/by$\mathrm{nc} / 4.0 /$.

\section{REFERENCES}

1. Fagin JA, Wells SA Jr. Biologic and clinical perspectives on thyroid cancer. $\mathrm{N}$ Engl $\mathrm{J}$ Med. 2016;375(11):1054-67.

2. Takami H, Ito Y, Okamoto T, Yoshida A. Therapeutic strategy for differentiated thyroid carcinoma in Japan based on a newly established guideline managed by Japanese Society of Thyroid Surgeons and Japanese Association of Endocrine Surgeons. World J Surg. 2011;35(1):111-21.

3. Passler C, Scheuba C, Prager G, et al. Prognostic factors of papillary and follicular thyroid cancer: differences in an iodine-replete endemic goiter region. Endocr Relat Cancer. 2004;11(1):131-9.

4. Haugen BR, Alexander EK, Bible KC, et al. 2015 American Thyroid Association Management guidelines for adult patients with thyroid nodules and differentiated thyroid cancer: the American Thyroid Association Guidelines Task Force on Thyroid Nodules and Differentiated Thyroid Cancer. Thyroid. 2016;26(1):1-133.
5. Kiyota N, Robinson B, Shah M, et al. Defining radioiodine-refractory differentiated thyroid cancer: efficacy and safety of lenvatinib by radioiodinerefractory criteria in the SELECT Trial. Thyroid. 2017;27(9):1135-41.

6. Durante C, Haddy N, Baudin E, et al. Long-term outcome of 444 patients with distant metastases from papillary and follicular thyroid carcinoma: benefits and limits of radioiodine therapy. J Clin Endocrinol Metab. 2006;91(8):2892-9.

7. Sabra MM, Sherman EJ, Tuttle RM. Tumor volume doubling time of pulmonary metastases predicts overall survival and can guide the initiation of multikinase inhibitor therapy in patients with metastatic, follicular cell-derived thyroid carcinoma. Cancer. 2017;123(15):2955-64.

8. Roman S, Lin R, Sosa JA. Prognosis of medullary thyroid carcinoma: demographic, clinical, and pathologic predictors of survival in 1252 cases. Cancer. 2006;107(9):2134-42.

9. Sugitani I, Miyauchi A, Sugino K, Okamoto T, Yoshida A, Suzuki S. Prognostic factors and treatment outcomes for anaplastic thyroid carcinoma: ATC Research Consortium of Japan cohort study of 677 patients. World J Surg. 2012;36(6):1247-54.

10. Kebebew E, Greenspan FS, Clark OH, Woeber KA, McMillan A. Anaplastic thyroid carcinoma treatment outcome and prognostic factors. Cancer. 2005;103(7):1330-5.

11. Matsui J, Yamamoto Y, Funahashi Y, et al. E7080, a novel inhibitor that targets multiple kinases, has potent antitumor activities against stem cell factor producing human small cell lung cancer H146, based on angiogenesis inhibition. Int $\mathrm{J}$ Cancer. 2008;122(3):664-71.

12. Okamoto K, Kodama K, Takase K, et al. Antitumor activities of the targeted multi-tyrosine kinase inhibitor lenvatinib (E7080) against RET gene fusion-driven tumor models. Cancer Lett. 2013;340(1):97-103.

13. Tohyama O, Matsui J, Kodama K, et al. Antitumor activity of lenvatinib (e7080): an angiogenesis inhibitor that targets multiple receptor tyrosine kinases in preclinical human thyroid cancer models. J Thyroid Res. 2014;2014:638747.

14. Schlumberger M, Tahara M, Wirth LJ, et al. Lenvatinib versus placebo in radioiodine-refractory thyroid cancer. N Engl J Med. 2015;372(7):621-30.

15. Kiyota N, Schlumberger M, Muro K, et al. Subgroup analysis of Japanese patients in a phase 3 study of lenvatinib in radioiodine-refractory differentiated thyroid cancer. Cancer Sci. 2015;106(12):1714-21. 
16. Takahashi S, Kiyota N, Yamazaki T, et al. A phase II study of the safety and efficacy of lenvatinib in patients with advanced thyroid cancer. Future Oncol. 2019;15(7):717-26.

17. Brose MS, Worden FP, Newbold KL, Guo M, Hurria A. Effect of age on the efficacy and safety of lenvatinib in radioiodine-refractory differentiated thyroid cancer in the phase III SELECT trial. J Clin Oncol. 2017;35(23):2692-9.

18. Eskens FA, Verweij J. The clinical toxicity profile of vascular endothelial growth factor (VEGF) and vascular endothelial growth factor receptor (VEGFR) targeting angiogenesis inhibitors; a review. Eur J Cancer. 2006;42(18):3127-39.

19. Keizer RJ, Gupta A, Mac Gillavry MR, et al. A model of hypertension and proteinuria in cancer patients treated with the anti-angiogenic drug E7080. J Pharmacokinet Pharmacodyn. 2010;37(4):347-63.

20. Keizer RJ, Gupta A, Shumaker R, Beijnen JH, Schellens JH, Huitema AD. Model-based treatment optimization of a novel VEGFR inhibitor. Br J Clin Pharmacol. 2012;74(2):315-26.

21. Hui EP, Ma BB, King AD, et al. Hemorrhagic complications in a phase II study of sunitinib in patients of nasopharyngeal carcinoma who has previously received high-dose radiation. Ann Oncol. 2011;22(6):1280-7.

22. Onoda N, Tokumoto M, Noda S, Ohira G, Kashiwagi S, Hirakawa K. A case of recurrent anaplastic thyroid cancer treated by lenvatinib after successful long-term multimodal therapy. J Jpn Surg Assoc. 2016;77(2):291-5.

23. Takahashi S, Kiyota N, Tahara M. Optimal use of lenvatinib in the treatment of advanced thyroid cancer. Cancers Head Neck. 2017;2:7.

24. Wirth LJ, Leboulleux S, Kiyota N, et al. Influence of tumor size and Eastern Cooperative Oncology
Group performance status (ECOG PS) at baseline on patient (pt) outcomes in lenvatinib-treated radioiodine-refractory differentiated thyroid cancer (RRDTC). J Clin Oncol. 2019;37(15):6081.

25. Akslen LA, Myking AO, Salvesen H, Varhaug JE. Prognostic importance of various clinicopathological features in papillary thyroid carcinoma. Eur J Cancer. 1992;29a(1):44-51.

26. Wu MH, Shen WT, Gosnell J, Duh QY. Prognostic significance of extranodal extension of regional lymph node metastasis in papillary thyroid cancer. Head Neck. 2015;37(9):1336-433.

27. D'Avanzo A, Treseler P, Ituarte PH, et al. Follicular thyroid carcinoma: histology and prognosis. Cancer. 2004;100(6):1123-9.

28. Gulcelik MA, Gulcelik NE, Kuru B, Camlibel M, Alagol H. Prognostic factors determining survival in differentiated thyroid cancer. J Surg Oncol. 2007;96(7):598-604.

29. Shah JP, Loree TR, Dharker D, Strong EW, Begg C, Vlamis V. Prognostic factors in differentiated carcinoma of the thyroid gland. Am J Surg. 1992;164(6):658-61.

30. Suzuki C, Kiyota N, Imamura Y, et al. Exploratory analysis of prognostic factors for lenvatinib in radioiodine-refractory differentiated thyroid cancer. Head Neck. 2019;41(9):3023-32.

31. Besic N, Hocevar M, Zgajnar J, Pogacnik A, GrazioFrkovic S, Auersperg M. Prognostic factors in anaplastic carcinoma of the thyroid-a multivariate survival analysis of 188 patients. Langenbecks Arch Surg. 2005;390(3):203-8.

32. Sugitani I, Kasai N, Fujimoto Y, Yanagisawa A. Prognostic factors and therapeutic strategy for anaplastic carcinoma of the thyroid. World J Surg. 2001;25(5):617-22. 